\title{
Über die Beziehung der Blutkatalase zur Sauerstoffkapazität des anämischen Bluts.
}

\author{
Von \\ Taiti Kumagai. \\ (熊谷太市) \\ (Aus dem Gerichtlich-Medizinischen Institut der \\ Kaiserlichen Tohoku-Universität zu Sendai. \\ Vorstand: Prof. Dr. T. Ishikawa.)
}

\section{Einleitung.}

Die kompensatorischen Vorgänge beim Sauerstoffmangel des Bluts bei Anämie sind, seit sie Boh $\mathrm{r}^{1)}$ schon im Jahre 1892 vorgebracht hatte, von vielen untersucht worden, und nur die Annahme, dass die Beschleunigung der Blutstromgeschwindigkeit zu obiger Kompensation" beitragen könnte, erkannten Plesch,") Weitzsäcker, ${ }^{3)}$ Morawitz, ${ }^{4)}$ Fahr, ${ }^{5)}$ Liljestrand ${ }^{6)}$ und Blalock ${ }^{7)}$ an.

Ishikawa und $\mathrm{Ki}^{8}$ ) stellten aber 1933 eine neue Betrachtung über die Beziehung der Blutkatalase zum Oxyhämoglobin an. Sie stellten nämlich auf Grund ihrer Versuchsergebnisse, wonach Oxyhämoglobin wenn dessen $p H$ und die Flüssigkeitsmenge der Blutkatalase am geeignetsten sind, die grösste Sauerstoffkapazität zeigt, die Behauptung auf, dass Katalase die Sauerstoffkapazität des Hämoglobins vergrössert.

Es ist mir auch gelungen, experimentell festzustellen, dass Blutkatalase die Sauerstoffkapazität des Oxyhämoglobins vermehrt und doch das reduzierte Glutathion sich nicht an dem Mechanismus beteiligt, hingegen das $p \mathrm{H}$ hat daran wichtige Teilnahme.

Die Frage der Anämie beschäftigte schon ältere Forscher, und zwar nach sehr verschiedenen Richtungen hin: was das $p H$ bei Anämie betrifft, so teilten Inoue $,^{9)} \mathrm{Gesell},{ }^{10)} \mathrm{Bald},{ }^{1}$ ' Rie gel, ${ }^{199} \mathrm{Hertz}$ mann ${ }^{13)}$ und andere dessen Abnahme mit; den $\mathrm{CO}_{2}$-Gehalt betreffend, berichteten auch schon früh $\mathrm{Jaksch},{ }^{14)}$ Peiper, ${ }^{15)} \mathrm{Kraus}$, ${ }^{16)}$ Rumpf, ${ }^{17)}$ Moranczenska, ${ }^{187}$ Brandenburg, ${ }^{19)}$ Waldvogel ${ }^{90)}$ und 
Zuntz, ${ }^{21)}$ später Bieling, ${ }^{22)}$ Milroy, ${ }^{\text {3) }}$ Cannon, ${ }^{24)}$ Barsky, ${ }^{25)}$ Penfield, ${ }^{26)}$ Lindemann, ${ }^{27)}$ Barr, ${ }^{28}$ Kuhlmann, ${ }^{29}$ Odaira, ${ }^{302}$ Gesell, ${ }^{31}$ Koeller, ${ }^{32}$ Wils o ${ }^{332}$ und andere über seine Verminderung bei perniziöser oder Aderlassanämie; ferner wurde Zunahme der Blutkatalase bei Anämie von Neumann, ${ }^{34)} \mathrm{Ochi}{ }^{35)}$ van Thienen ${ }^{36)}$ Nissen, ${ }^{37)}$ Korallus, ${ }^{38)}$ Levinger, ${ }^{39)}$ Segall, ${ }^{40)}$ Tögel, ${ }^{41 /}$ Bernstein, ${ }^{49}$ Berg, ${ }^{43)}$ Bode, ${ }^{44)}$ Krüger, ${ }^{45)}$ Bach und Kopássy ${ }^{46}$ und $\mathrm{Bach}^{47)}$ und Vermehrung des reduzierten Glutathions von Gabbe, ${ }^{48)}$ Waelsch,49) Platt ${ }^{50)}$ u. Valer $a^{51)}$ beobachtet; Butterfield, ${ }^{52)}$ Morawitz ${ }^{53)}$ und Masing ${ }^{54)}$ berichteten, dass Oxyhämoglobin bei Anämie keine Zunahme des Sauerstoffbindungsvermögens bewirkt.

Um nicht einzelnes, wie oben, sondern alles zusammen, Blutbilder, Blutkatalase, reduziertes Glutathion, Plasma- $p \mathrm{H}$ und $\mathrm{CO}_{2}$-Gehalt, am Blut ein und desselben anämischen Versuchstiers zu beobachten und dadurch ihren Zusammenhang untereinander zu untersuchen, stellte ich vorliegenden Versuch an.

\section{Experiment.}

\section{A. Methodik.}

Zum Versuch wurden täglich einmal mit reichlichem Tofukasu gefütterte Kaninchen verwandt, die in keinem Falle narkotisiert wurden. Um das Blut bei nur einmaligem Aderlass in grosser Menge zu entnehmen, wurden die Tiere am frühen Morgen mit ihren vier Pfoten an den Fixierungsapparat gefesselt und ihnen das Blut aus den Ohr- oder Jugularvenen entzogen. Diese ganze Behandlung nahm 30-40 Minuten in Anspruch. Mit dem Versuch begann ich am Tage des Aderlasses erst nach $2 \frac{1}{2}-3$ Ruhestunden nach der Behandlung. Der wiederholte Aderlass wurde immer an den Ohryenen ausgeführt. Toxische Anämie wurde dadurch hervorgebracht, dass man an vorhergehenden Tag den Tieren in die Rückengegend eine beschriebene Menge einer wässerigen Lösung von Phenylhydraz. mur. subkutan injizierte, und dann wurde am darauf folgenden Tage der Versuch angestellt. Das zu diesem Versuch benötigte Blut wurde ohne Ausnahme den Ohrvenen entnommen.

Bestimmung des Hämoglobingehalts: Fleischl-Mieschersches Verfahren wurde dazu angewendet.

Zählung der Erythrozyten: bei der Zählung der Erythrozyten wurde die Hay em sche Lösung und bei Leukozyten die Zürcksche verwandt; zur Zählung bediente ich mich der Thoma-Zeis sschen Zählkammer.

Lenkozytenbild: zur Färbung diente die May-Grünwaldsche und zur Doppelfärbung die Giemsa sche Lösung.

Bestimmung der Blutkatalase: Inouesches ${ }^{55}$ ) Verfahren wurde angewendet. 
Bestimmung des reduzierten Glutathions des Bluts: das Gabbesche ${ }^{56}$ ) Verfahren wurde benutzt.

Bestimmung des $\mathrm{CO}_{2}$-Gehalts: das Plasma wurde als Stoff genommen; die Bestimmung wurde nach der Mikromethode von Van Slyke und Cullen ${ }^{5 i}$ ) ausgeführt.

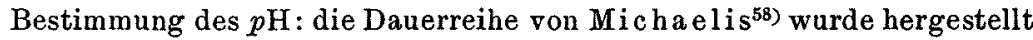
und das Plasma als Stoff genommen. Das beim Van Slykeschen Verfahren und zur Bestimmung des $p \mathrm{H}$ anzuwendende Plasma wird möglichst wenig der Luft ausgesetzt. wozu man das Blut aus den Ohrvenen des Tiers in ein mit Paraffinum fluidum gefülltes Reagenzglas hineintropfen lässt.

Bestimmung der Sauerstoffkapazität: wird durch den Barcroftschen ${ }^{59}$ Differentjalblutgasapparat bestimmt, wozu $1,0 \mathrm{ccm}$ Blut verwendet wird.

\section{B. Ergebnisse.}

\section{a. Akute Aderlassanämie.}

Die Katalaseindices zeigten am ersten Tage des Versuchs Abnahme im Vergleich zu denen vor dem Aderlass $(0,95 \rightarrow 0,86,0,93 \rightarrow 0,86$, $0,76 \rightarrow 0,68)$, aber etwa 24 Stunden nach dem Aderlass fast ausnahmslos Zunahme und zwar die höchsten Werte im ganzen Verlauf; von da an nahm er mit der Erholung von der Anämie im grossen und ganzen $a b$, wenn er auch noch mehr oder minder schwankte, und näherte sich allmählich dem normalen Wert, mitunter verminderte er sich nach 13-15 Tagen auch ein wenig unter diesen. Die Färbeindices waren am ersten Tage nach dem Aderlass unbestimmt und zeigten am zweiten Neigung zur Steigerung über den Wert vor dem Aderlass hinaus, aber am 4.-6. Tage sanken sie plötzlich, stiegen dann wieder und näherten sich schliesslich dem normalen Wert. Die Glutathionquotienten vermehrten sich schon am Tage des Aderlasses 6,69 $\rightarrow 7,24$, $5,98 \rightarrow 6,25$ u. $6,17 \rightarrow 8,13$, auch noch am folgenden Tag, ohne Ausnahme, um 1,79, 1,33, 1,74 u. 3,54, wobei sie den höchsten Wert zeigten, dann aber, mit der Erholung von der Anämie, nahmen sie immer ab und näherten sich schliesslich dem normalen Wert. Die Verhältnisse der Sauerstoffkapazitäten zu Hämoglobingehalte verminderten sich auch am Tage des Aderlasses, vermehrten sich aber am folgenden Tag ohne Ausnahme. Die $\mathrm{CO}_{\mathrm{z}}$-Gehalte zeigten am Tage des Aderlasses beträchtlich verminderte Werte, die aber schon am nächsten Tage wieder beinahe bis zum normalen Wert zurückkehrten; die Wiederherstellung trat viel schneller ein als bei Hämoglobin: nach 3-4 Tagen stiegen die Wert schon über den normalen. Die Plasma- $p H$ zeigten einen ähnliches Verlauf: am ersten Tage des Aderlasses ver- 


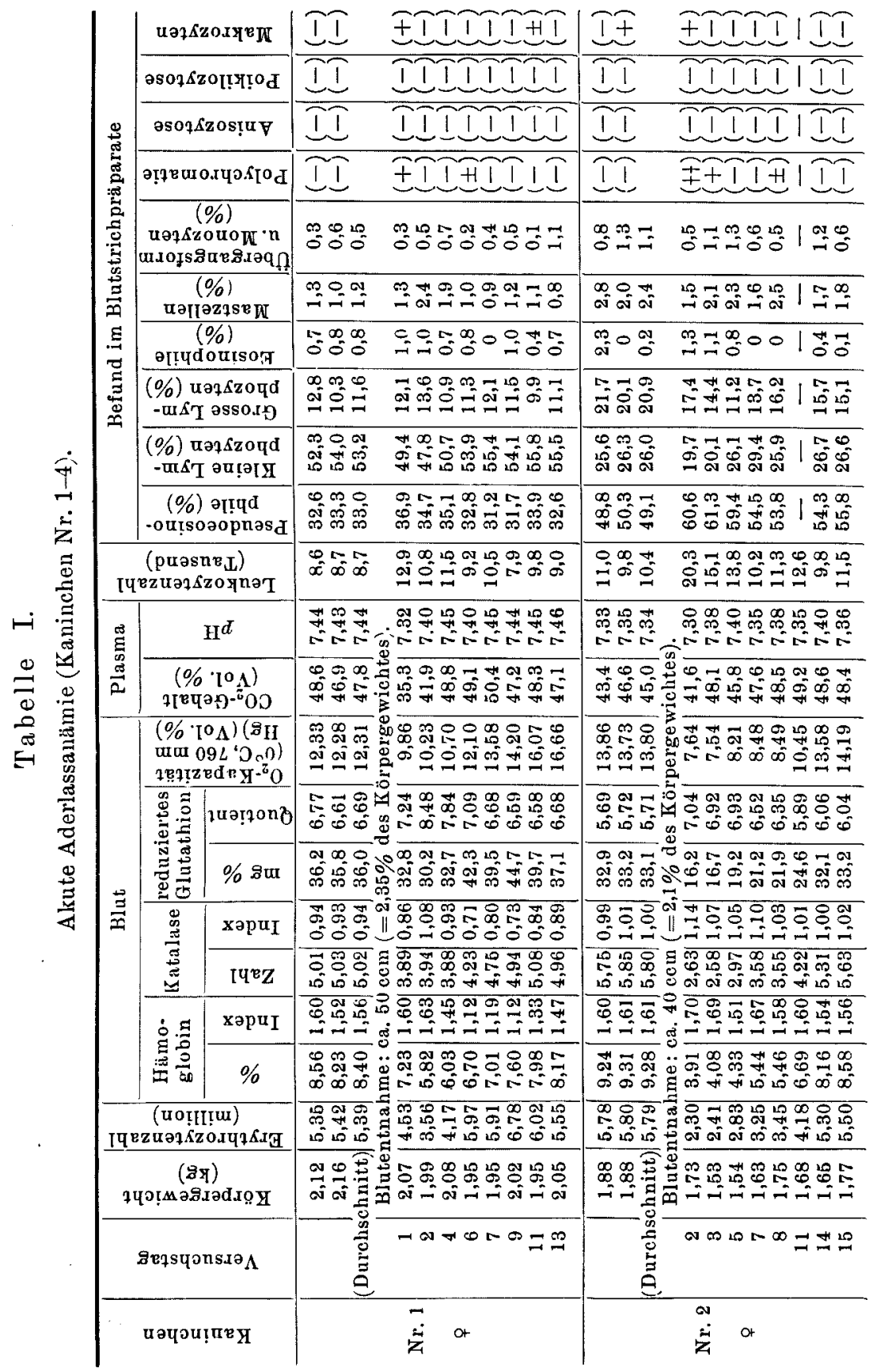




\begin{tabular}{|c|c|c|c|c|c|c|}
\hline \multirow{10}{*}{ 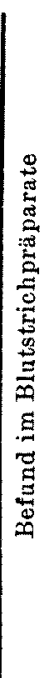 } & \multicolumn{2}{|c|}{ 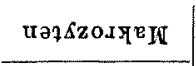 } & IH & $\pm 11 \pm 111$ & 11 & 111111 \\
\hline & \multicolumn{2}{|c|}{ อso7\$zOI!Y!O d } & II & IIIIIII & 11 & IIIIII \\
\hline & \multicolumn{2}{|c|}{ әso7凡zosłu } & $i$ & IIIIIII & II & IIIIII \\
\hline & \multicolumn{2}{|c|}{ 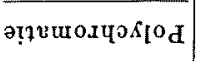 } & II & IIH|IIH & II & सITHII \\
\hline & \multicolumn{2}{|c|}{ 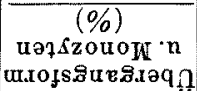 } & $\sigma=$ & 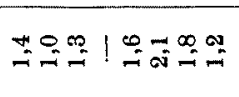 & $\begin{array}{l}0 \\
0 \\
0\end{array}$ & 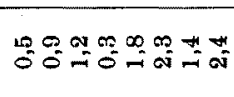 \\
\hline & \multicolumn{2}{|c|}{$\begin{array}{c}(\%) \\
\text { uəIIวz?se II } \\
\end{array}$} & $\underline{2}=$ & 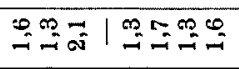 & $\cong 9$ & 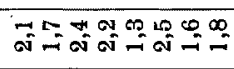 \\
\hline & \multicolumn{2}{|c|}{$\begin{array}{c}1 \%) \\
\text { गा!परou!sog }\end{array}$} & 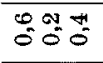 & $g=10000$ & $0 \stackrel{9}{0} \stackrel{\mathfrak{s}}{\sigma}$ & 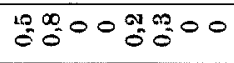 \\
\hline & \multicolumn{2}{|c|}{ 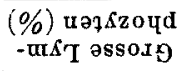 } & 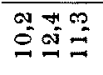 & 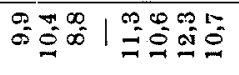 & $\vec{\infty}=0$ & 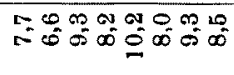 \\
\hline & \multicolumn{2}{|c|}{ 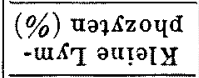 } & $\begin{array}{l}\infty \\
0 \\
0 \\
0\end{array}$ & 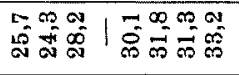 & 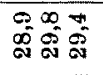 & 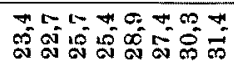 \\
\hline & \multicolumn{2}{|c|}{$\begin{array}{c}\%) \text { əเ!Yd } \\
\text {-ou!soəopnəs d }\end{array}$} & 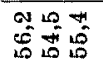 & 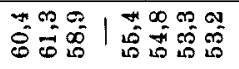 & 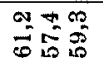 & 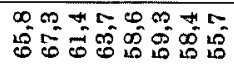 \\
\hline \multicolumn{3}{|c|}{ 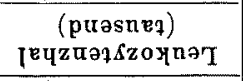 } & $\begin{array}{ll}0 & \infty \\
\infty & \infty\end{array}$ & 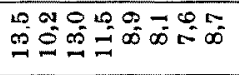 & $\begin{array}{lll}\pi & 0 \\
0 & 0 & 0\end{array}$ & 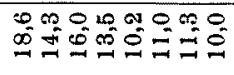 \\
\hline \multirow{2}{*}{ 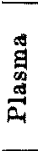 } & \multicolumn{2}{|c|}{$\mathbf{H}^{d}$} & 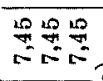 & 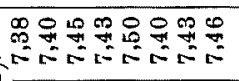 & 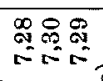 & 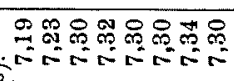 \\
\hline & \multicolumn{2}{|c|}{ 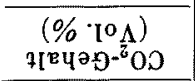 } & 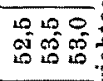 & 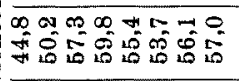 & 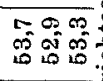 & 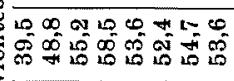 \\
\hline \multirow{7}{*}{ 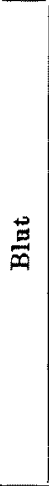 } & \multicolumn{2}{|c|}{ 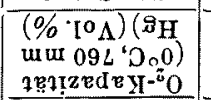 } & 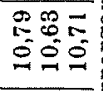 & 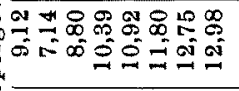 & 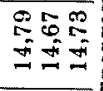 & 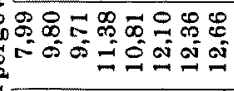 \\
\hline & \multirow{2}{*}{ 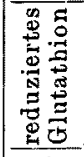 } & นนวเุดกซ & 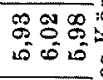 & 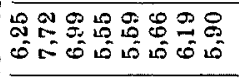 & 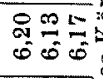 & $\begin{array}{l}m=0 \\
m \infty \\
\infty \\
\infty\end{array}$ \\
\hline & & $\% 8 \mathrm{ur}$ & 业 & 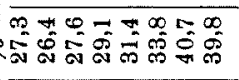 & 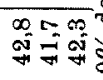 & 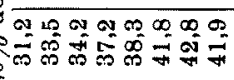 \\
\hline & \multirow{2}{*}{ 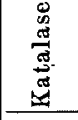 } & xәpuI & 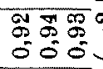 & 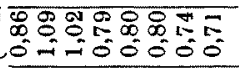 & 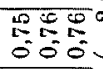 & 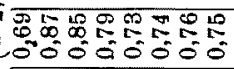 \\
\hline & & Іบ $Z Z$ & 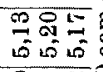 & 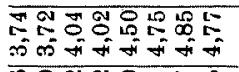 & $=\frac{\infty}{10}=\frac{\infty}{20}$ & 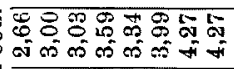 \\
\hline & \multirow{2}{*}{ 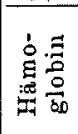 } & хәри I & 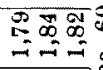 & 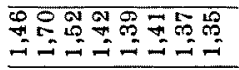 & 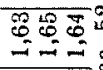 & 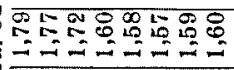 \\
\hline & & $\%$ & $\begin{array}{l}8.00 \\
80.0 \\
000\end{array}$ & 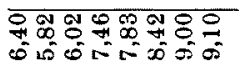 & 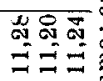 & 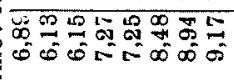 \\
\hline \multicolumn{3}{|c|}{ 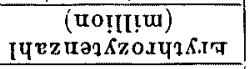 } & 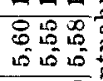 & 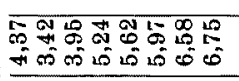 & $\begin{array}{lll}1 & \\
8 & 0 & 0 \\
0 & 0 \\
0 & 0 & 0 \\
\end{array}$ & 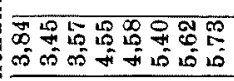 \\
\hline \multicolumn{3}{|c|}{ 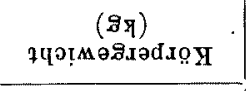 } & 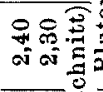 & 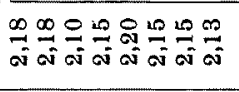 & 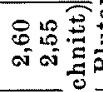 & 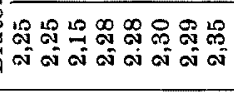 \\
\hline \multicolumn{3}{|c|}{ SEารYonsir $\Lambda$} & 总 & $\because \infty+\infty \infty O \pi 0$ & 总 & 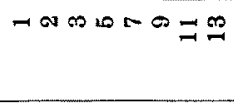 \\
\hline \multicolumn{3}{|c|}{ 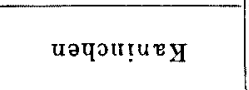 } & & $\ddot{\dot{z}} \leftrightarrow$ & & $\ddot{\vec{z}}$ \\
\hline
\end{tabular}


mehrten sie sich $7,44 \rightarrow 7,32,7,45 \rightarrow 7,38$ u. $7,29 \rightarrow 7,19$, am 2 . Tage nahmen sie nur noch wenig zu; also ein ziemlich viel langsamerer Wechsel als bei dem $\mathrm{CO}_{2}$-Gehalt. Dementsprechend trat auch bei dieser vorübergehenden Aderlassanämie infolge grösseren Blutverlusts Neigung zur Azidosis auf, die stets in Beziehung zur Menge des verlorenen Bluts steht, aber ihr Verhältnis auf die Dauer konnte nicht genauer festgestellt werden. Die Leukozyten vermehrten sich mit dem Blutverlust, nahmen aber mit der Erholung von der Anämie wieder ab. Polychromatie und Makrozytose, welche öfters als Ursache ganzer Kompensationsvorgänge bei dieser Anämie bestritten wurden, traten bei meinem Versuche nicht so viel auf, wie sie solche Funktionen hätten ausüben können. Die Leukozytenbilder deuteten bei jedem Falle auf Neigung zur Abnahme der Lymphozyten und zur Zunahme der pseudoeosinophilen bzw. eosinophilen Zellen.

b. Äkute toxische Anämie durch Phenylhydrazin.

Bei einmaliger toxischer Anämie infolge grösserer Giftmenge zeigten die Färbeindices ohne Ausnahme eine Verminderung $1,6 \mathrm{C} \rightarrow$ $1,51,1,46 \rightarrow 1,40,1,68 \rightarrow 1,56$ u. $1,44 \rightarrow 1,36$; in 4-6 Tagen erreichte die Abnahme ihren höchsten Grad, woraus zu erkennen ist, dass das betreffende Gift das Hämoglobin früher zerstört als die Erythrozyten, und das Hämoglobin manchmal am 6.-8. Tage wieder normal zu werden beginnt, die Erythrozyten jedoch noch nicht; jenes kehrte sehr schnell wieder zur Norm zurück.

Die Katalaseindices fingen ohne Ausnahme zu steigen an und zeigten am 4.-6. Tage, wenn die Erythrozyten am stärksten abnahmen, meistens ihre höchsten Werte, die mehr als 1,5-2 mal so gross waren wie der normale, und dann näherten sie sich allmählich dem Normalwert. Die Glutathionquotienten erreichten auch am 4.-6. Tage ihre höchsten Werte, die mehr als 2-3 mal so gross wie der normale waren, und zeigten wenigstens noch am 15.-17. Tage Zunahme um 1-4\%. Aber die Sauerstoffkapazität verhielt sich bei der durch methämoglobinbildendes Gift verursachten toxischen Anämie ganz anders als bei der Aderlassanämie. Am Tage nach der Injektion (ungefähr 14-15 Stunden nach Injektion) nahm das Blut selbst eine dunkelrote Färbung an, die auch nicht ins Scharlachrote überging, wenn es zur Sättigung des Sauerstoffs geschüttelt wurde, und schon makroskopisch war Abnahme des Oxyhämoglobins und Bildung von Methämoglobin zu sehen. Die Verhältnisse der Sauerstoffkapazitäten zu Hämoglobingehalte verminderten sich beträchtlich, bis unter die 


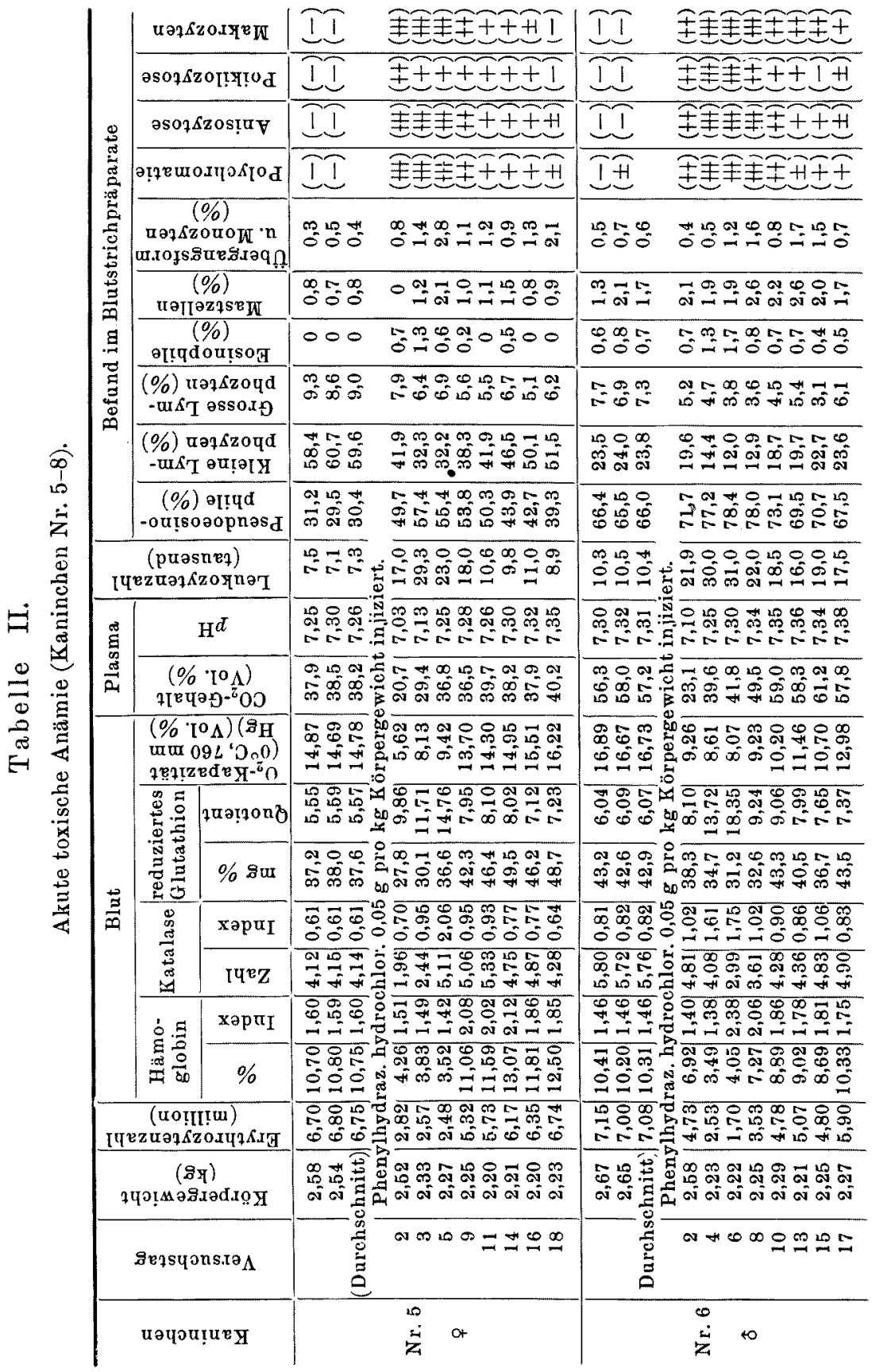




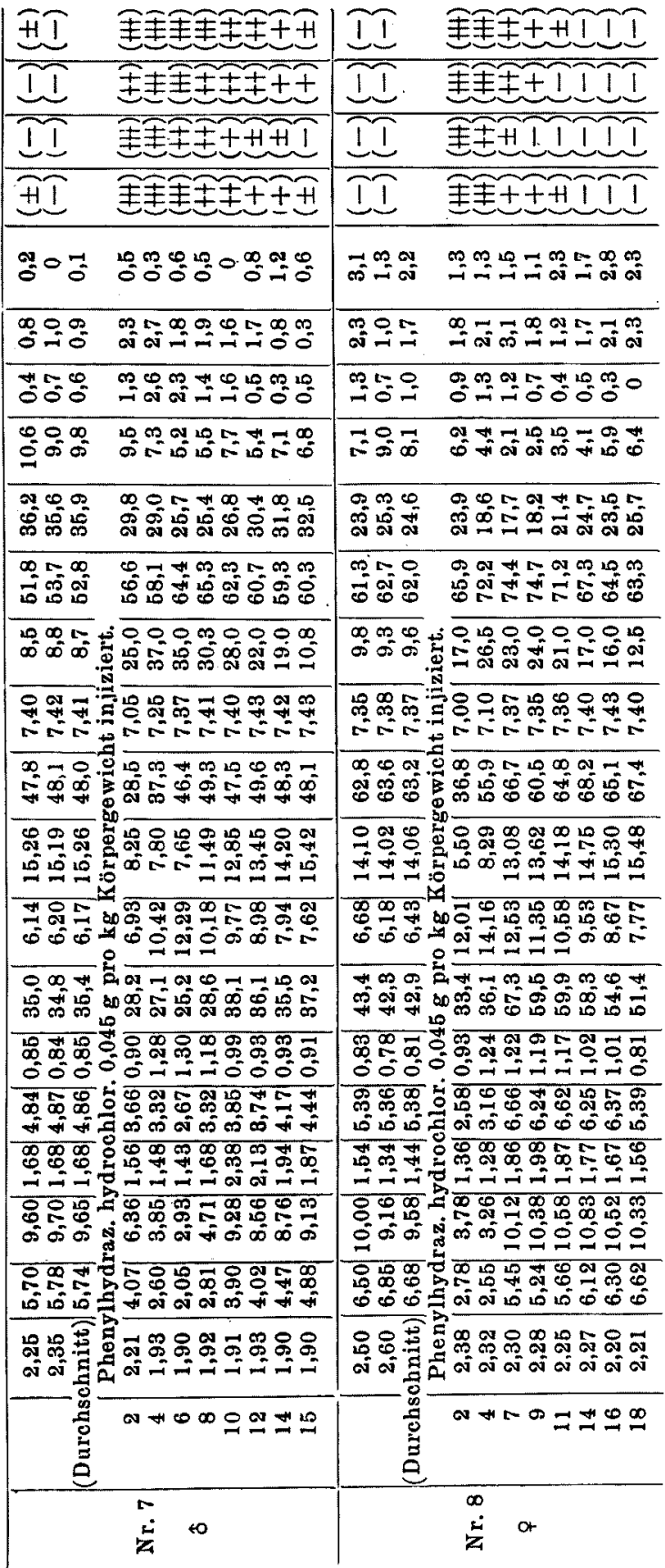

normalen Werte; aber ungefähr am 4. Tage fingen die Kapazitäten plötzlich an, lebhaft zuzunehmen, und deshalb stiegen die betreffende Verhältnisse schon am 4.-8. Tage auf die höchsten Werte und kamen dann am 15.-17. Tage wieder den Normalwerte sehr viel näher. Die $\mathrm{CO}_{2}$ Gehalte verhielten sich auch anders als beim vorhergehenden Versuche: ihr Verminderungen waren grösser als die der Hämoglobin; sie verminderten sich nämlich am ersten Tage des Versuchs sehr beträchtlich, wobei ausgeprägte Azidosis eintrat; die Veränderungen liefen mit den der Hämoglobin nicht parallel und erreichten nach der Injektion mit der Zeit wieder fast die Anfangswerte; diese 
Wiederherstellungen erfolgten schon in 6-10 Tagen, viel früher als bei Hämoglobin. Die Plasina- $p \mathrm{H}$ veränderte sich übereinstimmend mit der Abnahme der $\mathrm{CO}_{\mathrm{a}}$-Gehalte, nur in geringerem Grade. Das Versuchstier hatte bei höchster Azidosis gar keinen Appetit, und seine Bewegung war auch aufs äusserste beschränkt. Die Plasma$p \mathrm{H}$ schwankte fast parallel mit den $\mathrm{CO}_{2}$-Gehalten, sie stellten sich ziemlich viel langsamer als bei der Aderlassanämie wieder her, was davon herrübren könnte, dass auf das Tier zwei Einflüsse, nicht nur Anämie, sondern auch Gift, einwirkten. Bei dieser toxischen Anämie zeigten die Erythrozyten nicht am Tage nach der Injektion, sondern vielmehr erst am 4.-6. Tage ilıren niedrigsten Wert, und fast gleichzeitig vermehrten sich die Leukozyten. Diese zeigten klar das Bild perniziöser Anämie, wobei alle Blutzellen auftraten. Die Abnahme der Lymphozyten und die Zunahme der pseudoeosinophilen und eosinophilen Zellen waren besonders beträchtlich.

Beim Vergleich der Verhältnisse der Erythrozyten, des Hämoglobins und des Färbeindexes bei Aderlassanämie mit denen bei toxischer Anämie ist ein ausgeprägter Unterschied zu erkennen. Bei der Aderlassanämie ist die Wiederherstellung des Hämoglobins schwächer und später als die der Erythrozy ten; bei der toxischen Anämie ist es umgekehrt: die Zunahme und die Wiederherstellung des Hämoglobins gehen manchmal der der Erythrozyten vorher, wobei der Grad oder die Geschwindigkeit bei jenem immer den oder die bei Erythrozyten übertrifft.

\section{c. Anämie durch wiederholte Aderlässe.}

Bei Anämie infolge wiederholter Aderlässe zeigten die Färbeindices am Tage nach dem letzten Aderlass $1,6 \mathrm{C} \rightarrow 1,68,1,80 \rightarrow 1,98$, $2,03 \rightarrow 2,381,65 \rightarrow 1,98$, also die höchsten Werte.

Die Katalaseindices stiegen bei jeder Wiederholung der Aderlasses fast ohne Ausnahme : $0,76 \rightarrow 0,88,0,86 \rightarrow 0,99,1,11 \rightarrow 1,34,0,91 \rightarrow$ 1,$42 ;$ nach dem Aufhören des letzten Aderlasses nahmen sie auch noch zwei Tage lang ziemlich zu, dann aber verminderten sie sich allmählich und näherten sich dem Normalwerte. Die Glutathionquotienten nahmen mit der Wiederholung des Aderlasses ebenfalls allmählich zu : $6,25 \rightarrow 8,56,7,04 \rightarrow 8,85,6,01 \rightarrow 8,14,5,74 \rightarrow 9,80$; auch nach dem Aufhören des Aderlasses stiegen sie noch 2-4 Tage lang immer weiter. Die Verhältnisse der Sauerstoffkapazitäten zu Hämoglobin stiegen 


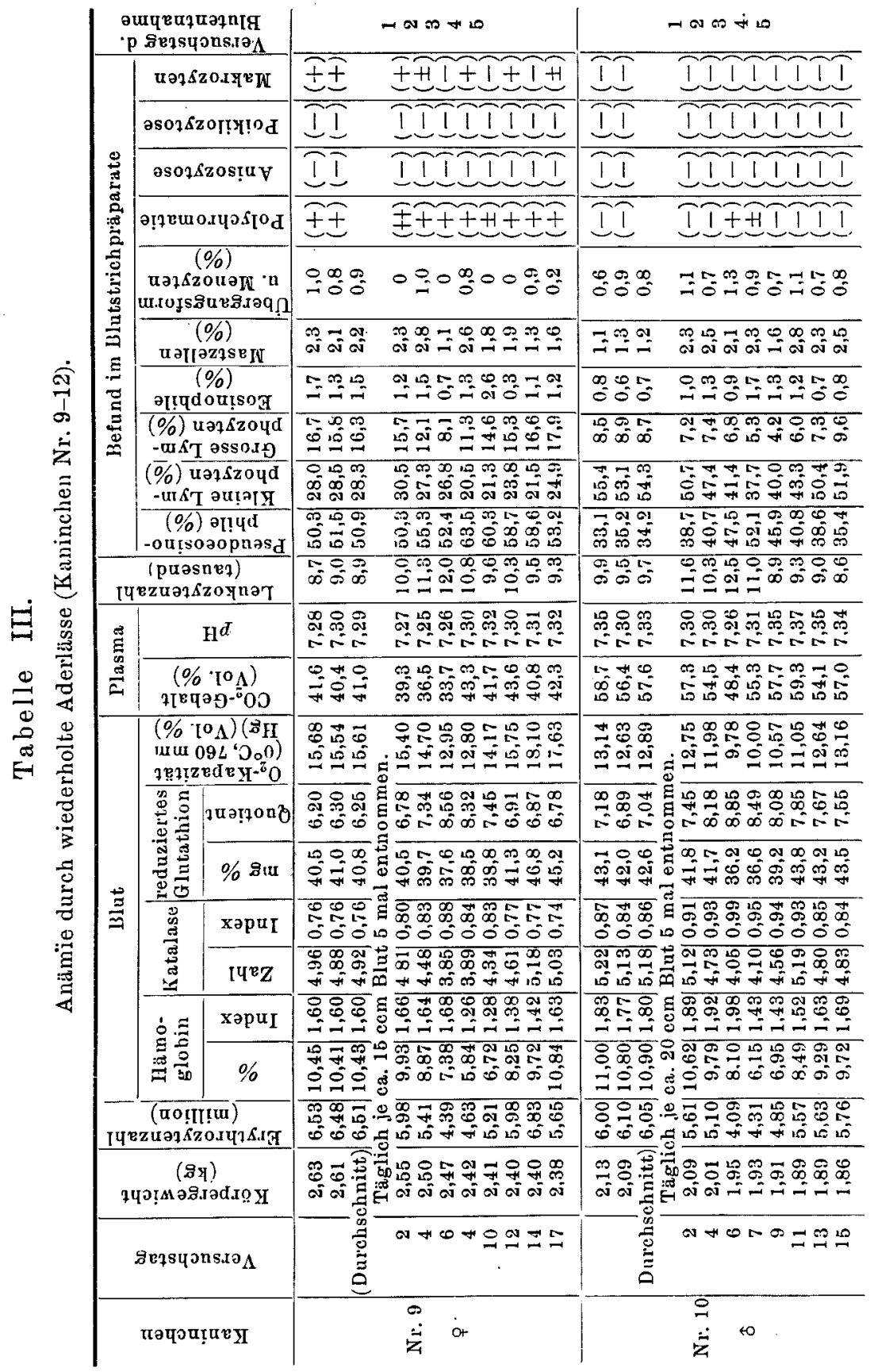




\begin{tabular}{|c|c|c|c|c|}
\hline \multicolumn{3}{|c|}{ 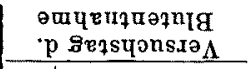 } & $-\infty \infty x+\infty$ & 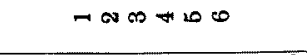 \\
\hline \multirow{10}{*}{ 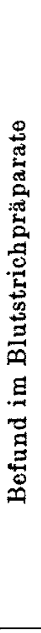 } & \multicolumn{2}{|c|}{ 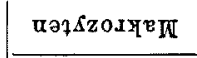 } & $11 \pm+1+1$ & IEt土IE \\
\hline & \multicolumn{2}{|c|}{ өsoๆkzol!Y!०d } & IIIIIII & IIIII \\
\hline & \multicolumn{2}{|c|}{ əso१Szos!ut } & IIIIITI & TITTT \\
\hline & \multicolumn{2}{|c|}{ ә!า } & $11++111+$ & $T+T+T H T$ \\
\hline & \multicolumn{2}{|c|}{ 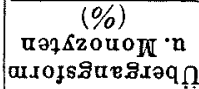 } & 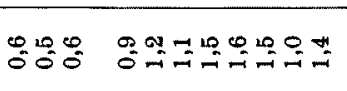 & $\begin{array}{lll}0 & 00 & 0 \\
0 & 0 & 0\end{array}$ \\
\hline & \multicolumn{2}{|c|}{$\begin{array}{c}(\%) \\
\text { पәIอzqsed }\end{array}$} & 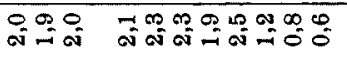 & 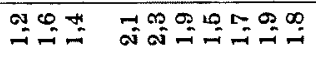 \\
\hline & \multicolumn{2}{|c|}{$\begin{array}{c}(\%) \\
\text { әा!पdoutsorg }\end{array}$} & 영영 & 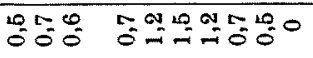 \\
\hline & \multicolumn{2}{|c|}{ 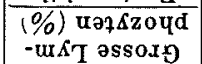 } & 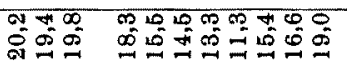 & 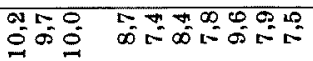 \\
\hline & \multicolumn{2}{|c|}{ 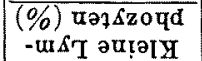 } & 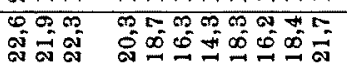 & 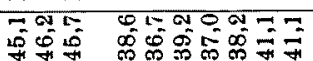 \\
\hline & \multicolumn{2}{|c|}{$\begin{array}{c}\text { (\%) əItyd } \\
\text {-outsozopnes }\end{array}$} & 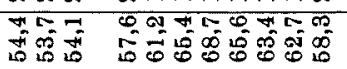 & 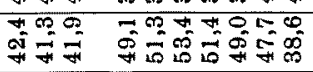 \\
\hline \multicolumn{3}{|c|}{ 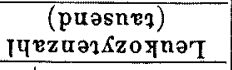 } & 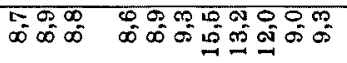 & 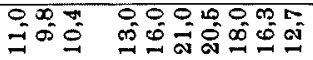 \\
\hline \multirow{2}{*}{ 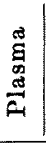 } & \multicolumn{2}{|r|}{$\mathbf{H}^{d}$} & 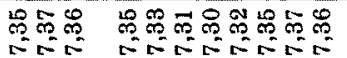 & 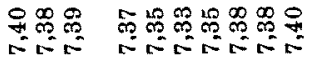 \\
\hline & \multicolumn{2}{|c|}{ 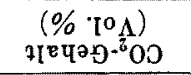 } & 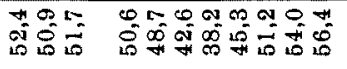 & 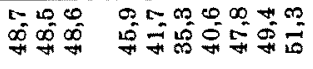 \\
\hline & \multicolumn{2}{|c|}{ 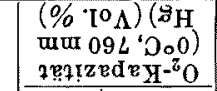 } & 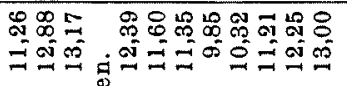 & 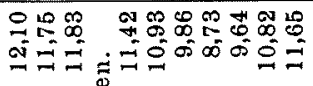 \\
\hline & \multirow{2}{*}{ 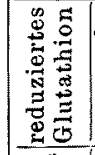 } & puẹpong & 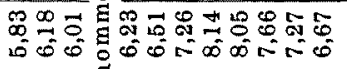 & 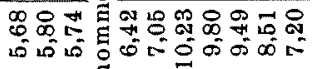 \\
\hline & & $\%$ sux & 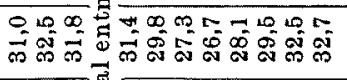 & 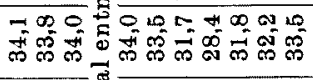 \\
\hline$\underline{\Xi}$ & \multirow{2}{*}{ 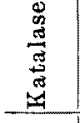 } & хәри I & 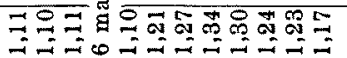 & 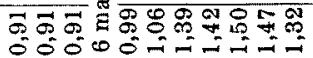 \\
\hline & & [पе Z & 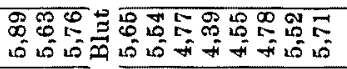 & 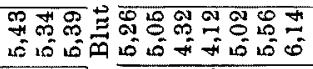 \\
\hline & \multirow{2}{*}{ 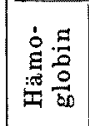 } & хәриі & 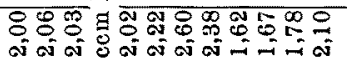 & 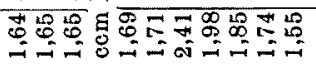 \\
\hline & & $\%$ & 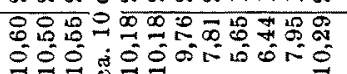 & 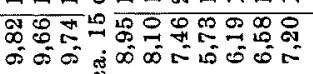 \\
\hline \multicolumn{3}{|c|}{ 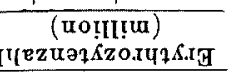 } & 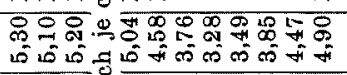 & 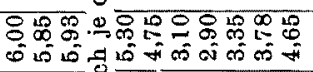 \\
\hline \multicolumn{3}{|c|}{ 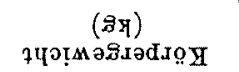 } & 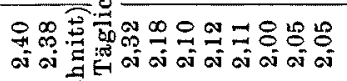 & 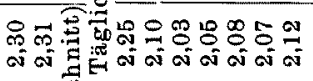 \\
\hline \multicolumn{3}{|c|}{ 8̈eวsyons.ta $\Lambda$} & $m * 0 \times 0=$ & $\infty+\infty-\infty= \pm$ \\
\hline \multicolumn{3}{|c|}{ 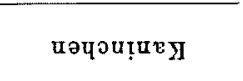 } & & $\stackrel{\stackrel{N}{z}}{\dot{\vec{z}}}$ \\
\hline
\end{tabular}


auch mit jeder Wiederholung des Aderlasses allnählich. Die $\mathrm{CO}_{2}$ Gehalte nahmen dagegen mit der Wiederholung des Aderlasses allmählich ab; diese Verminderung waren geringer als bei Hämoglobin. Bei Anämie infolge wiederholter Aderlässe verliefen die Verminderungen der Erythrozyten also fast geradlinig, die der $\mathrm{CO}_{\mathrm{g}}$-Gebalte hingegen noch langsamer, ja diese neigten sogar allmählich zur Azidosis. Daraus kann man ersehen, dass tägliche Wiederholung des Aderlasses in einer bestimmten Menge schliesslich auch tödliche Azidosis verursacht. Die Plasma- $p H$ nahmen mehr oder minder ab, nur nicht so beträchtlich wie die $\mathrm{CO}_{2}$-Gehalte; wenn man nur die $\mathrm{Ver}$ änderung des Plasma- $p \mathrm{H}$ beobachtet und die Bestimmung des $\mathrm{CO}_{2}-\mathrm{Ge}$ halts unterlässt, so kann der zur Azidosis neigende Zustand ganz unbemerkt bleiben. Die Leukozyten zeigten dasselbe Bild wie das oben erwähnte. Die Veränderungen dieser Werte im Blut bei akuter oder wiederholter Aderlassanämie hangen im grossen und ganzen von der durch den Aderlass verlorenen Blutmenge ab.

\section{d. Anämie durch wiederholte Phenyl- hydrazininjektionen.}

Bei der Beobachtung der Beeinflussung durch wiederholte Giftinjektion wurde bemerkt, dass sich Hämoglobin in höherem Grade verminderte als die Erythrozyten beeinflusst wurden, Hämoglobin verminderte sich nämlich während wiederholt ausgeführter Injektionen sehr beträchtlich, aber nach ihrem Aufhören vermehrten sie sich lebhaft und in hohem Grade. Daher folgten sich die Färbeindices bei jedem Falle folgendermassen; $1,63 \rightarrow 1,27 \rightarrow 2,83,1,57 \rightarrow 1,20$ $\rightarrow 2,64,1,79 \rightarrow 1,04 \rightarrow 2,39,1,86 \rightarrow 1,36 \rightarrow 2,31$, dem Verlauf bei der Anämie infolge wiederholten Aderlasses gerade entgegengesetzt.

Die Steigerungen der Katalaseindices waren auch sehr bedeutend $1,12 \rightarrow 1,86,0,82 \rightarrow 1,71,0,86 \rightarrow 1,53,0,78 \rightarrow 1,17$; dabei aber waren die Erhöhungen nicht immer gleichmässig, sondern ziemlich schwankend. Die Glutathionquotienten stiegen : $7,84 \rightarrow 14,35,4,89 \rightarrow 14,60$, $7,16 \rightarrow 15,87,7,03 \rightarrow 15,36$. Diese lebhafte Zunahme machte den Eindruck, als ob ihr Wert durch weiter fortgesetzte Wiederholung der Injektion, wofern das Tier dadurch nicht sterben würde, mehrere Male, ja zehn-, zwanzig- und dreissigmal usw. so hoch steigen würde wie der normale Wert. Die Verhältnisse der Sauerstoffkapazitäten zu Hämoglobin sanken am Tage nach der Injektion zunächst unter 


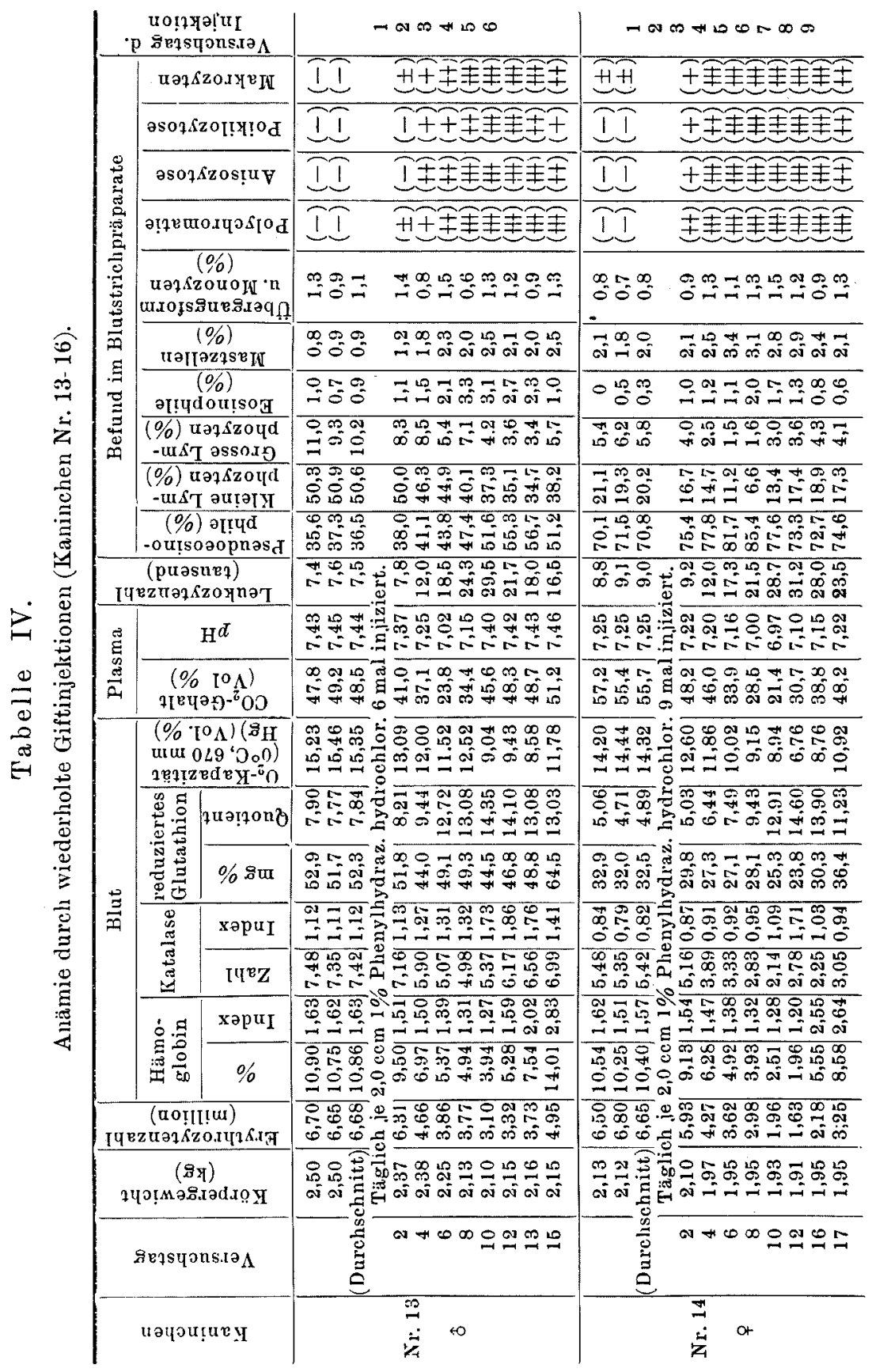




\begin{tabular}{|c|c|c|}
\hline \multicolumn{2}{|c|}{ 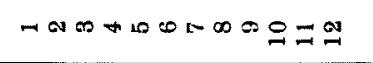 } & - \\
\hline II & 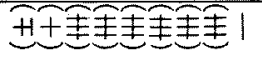 & II I士王主主主 \\
\hline$\sqrt{1}$ & 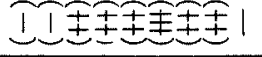 & II II王王主王 \\
\hline II & 开琶主主主主 & II 开玨主主主 \\
\hline$\sqrt{1}$ & 计士主主主主主| & 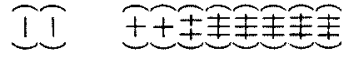 \\
\hline$=08$ & 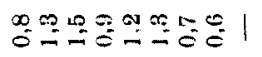 & 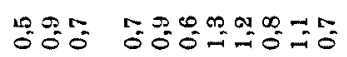 \\
\hline Lis ô को & 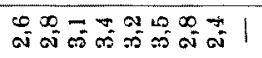 & 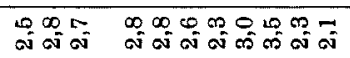 \\
\hline$g_{0}^{m} \circ g_{0}^{\infty}$ & 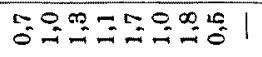 & 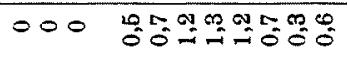 \\
\hline 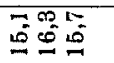 & 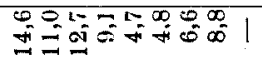 & mog \\
\hline 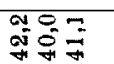 & 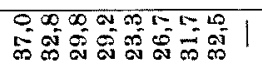 & 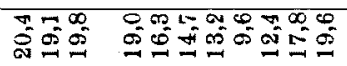 \\
\hline 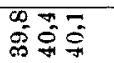 & 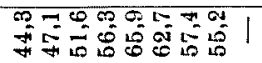 & 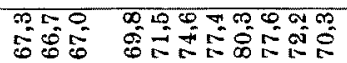 \\
\hline $\begin{array}{ll}\infty \\
0 \\
0=0\end{array}$ & 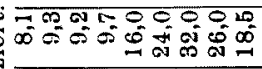 & 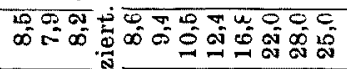 \\
\hline 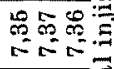 & 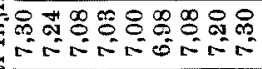 & 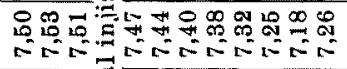 \\
\hline 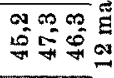 & 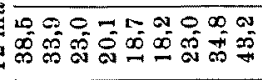 & 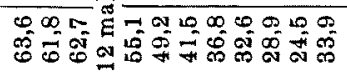 \\
\hline 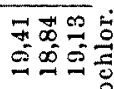 & 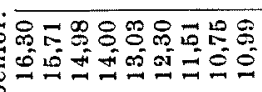 & 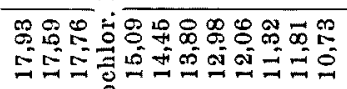 \\
\hline 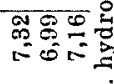 & 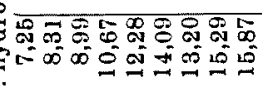 & 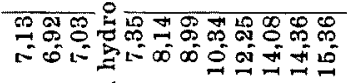 \\
\hline 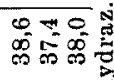 & 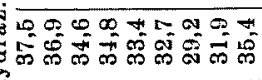 & 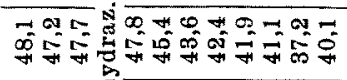 \\
\hline 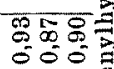 & 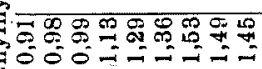 & 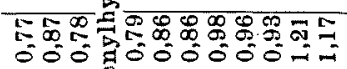 \\
\hline 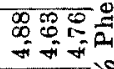 & 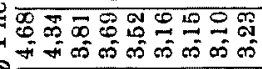 & 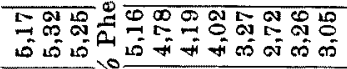 \\
\hline$\stackrel{\infty}{=} \stackrel{0}{=}$ & 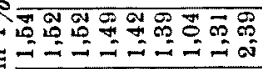 & 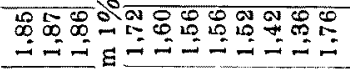 \\
\hline 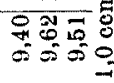 & 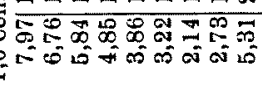 & 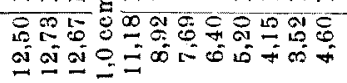 \\
\hline 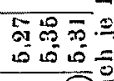 & 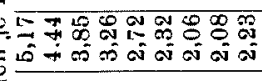 & 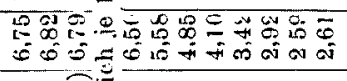 \\
\hline 我 & 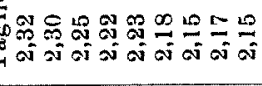 & 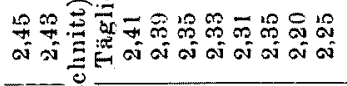 \\
\hline 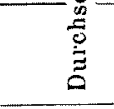 & $a+\infty \infty \equiv N=\infty$ & $\omega * \bullet \infty \subseteq \cong \pm 0$ \\
\hline & $\stackrel{0}{\dot{z}}$ & $\frac{0}{\dot{z}}$ 世 \\
\hline
\end{tabular}

den Normalwerte, ganz wie bei der einmaligen Injektion einer grossen Menge, aber allmählich nahmen sie dann wieder zu. Die $\mathrm{CO}_{2}$-Gehalte nahmen allmählich ab, und mit jeder Wiederholung der Injektion wurde immer stärkere Azidosis hervorgerufen. Das Tier hatte bei diesem Verlauf wenig Appetit und bewegte sich auch nur träge, und sein Plasma trubte sich ziemlich stark, eine Veränderung; die auch makroskopisch zu erkennen wax. Die Plasma- $p H$ verminderten sich auch allmählich und zwar so beträchtlich, lass der 14. Fall dadurch zum sterbenden Zustande gebracht wurde. Die Bilder der Leukozyten im Blut stimmten fast mit den vorhergehenden überein. 


\section{Zusammenfassung und Besprechung.}

Ich erwähnte in der Einleitung, dass die Verminderung des $\mathrm{CO}_{2}$ Gehalts bei Anämie entscheidend ist; dagegen behaupteten Lö w y ${ }^{60}$ Strauss, ${ }^{61)}$ Evans $^{62)}$ u. Saito ${ }^{63)}$ ihre Zunahme und Fitzgerald ${ }^{(4)}$ berichtete, dass sie normal bleibe. Tatum ${ }^{65)}$ und $\mathrm{Bennet}{ }^{66)}$ beobachteten, dass bei Aderlassanämie der $\mathrm{CO}_{2}$-Gehalt abnahm, jedoch am folgenden Tag wieder auf den Normalwert stieg. Der von mir ausgeführte Versuch aber ergab, dass bei Aderlassanämie die Verminderung des $\mathrm{CO}_{2}$-Gehalts wenigstens innerhalb 3 Stunden nach dem Aderlass sehr beträchtlich war und auch am folgenden Tag der normale Wert noch nicht oder kaum erreicht wurde. Daraus darf man schliessen, dass bei der Aderlassanämie jede Wiederholung des Aderlasses immer stärkere Azidosis hervorrufen kann. Bei der toxischen Anämie tritt besonders heftige Azidosis ein. Wie verhält sich nun das Plasma- $p \mathrm{H}$, welches in enger Beziehung zum $\mathrm{CO}_{2}$-Gehalt steht?

- Means $\mathrm{s}^{(7)}$ behauptete auf Grund seiner klinischen Beobachtung des Säure-Alkali-Gleichgewichts beim Blut verschiedener Anämien, dass das Blut- $p \mathrm{H}$ sich niemals vermindere. Evans beobachtete bei der Bestimmung des Blut- $p \mathrm{H}$ zweier durch Urethan narkotisierter Katzen nach dem Blutverlust bei einem Tier Abnahme, beim anderen jedoch Zunahme. Bennet berichtete, dass das Blut- $p \mathrm{H} 4-5$ Stunden nach dem Blutverlust abnehme, am folgenden Tag aber sich auf der Alkaliseite befinde und in 2-3 Tagen wieder den normalen Wert erreiche. Aber ich beobachtete, dass innerhalb 3 Stunden nach dem Blutverlust das Plasma- $p \mathrm{H}$ abnahm und am folgenden Tag, obgleich es bei einigen Fällen sich noch mehr auf die Alkaliseite wendete, meistens einen etwas niedrigeren Wert als den normalen zeigte. Daran erkenne ich, dass der Blutverlust auch die Abnahme des Plasma$p \mathrm{H}$ beeinflusst. Es wäre nun zu untersuchen, wodurch diese Verminderung des $\mathrm{CO}_{2}$-Gehalts und des Plasma- $p \mathrm{H}$ verursacht wird.

Nach Hender's on $\mathbf{s}^{(8)}$ Ansicht nehme das Hämoglobin infolge der Anämie ab, wodurch Sauerstoffmangel erzeugt werde; dieser reize das Atemzentrum, was U̇berventilation bewirke; dadurch wiederum werde das Blut in hohem Grade alkalisch ; damit der Normalwert der Alkalität erhalten werde, wtirde Nat. bicarb. ins Gewebe durchgelassen, aber infolge des Durchlasses der im Gewebe gebildeten Säure ins Blut nicht neutralisiert. Nach Bennet werde dagegen das Blut bei Blutverlust nach etwa 30 Minuten sauer und das Blut- $p \mathrm{H}$ nehme während mehrerer Stunden ab, aber nicht an ein Eindringen des Salzes 
aus dem Blut ins Gewebe, damit das Blut-pH abnehmen könne, sei nicht zu denken, obwohl auf den Blutverlust augenblicklich Überventilation eintrete; auch wenn es geschehen könnte, so wäre solch Durchlass doch nicht bedeutend genug, um das Blut- $p \mathrm{H}$ zu vermindern. Aber nach dem Ergebnjs des von mir ausgeführten Versuchs ist die Verminderung des $\mathrm{CO}_{2}$-Gehalts grösser als die des Blut- $p \mathrm{H}$; daraus könnte man mit Recht schliessen, dass bei Anämie die durch abnorme und unpassende Oxydation gebildete Säure aus dem Gewebe ins Blut infundiert wird und der $\mathrm{CO}_{2}$-Gehalt zunächst darauf einwirkt, dass dann aber, wenn diese Einwirkung unmöglich wird, gerade dadurch das $p \mathrm{H}$ vermindert wird. Also trete ich der Ansicht Bennets bei. Bei der toxischen Anämie wird die Oxydation im Blut durch das Eindringen des Blutgifts, d. h. des Phenylhydrazins (Allen), ${ }^{69}$ in hohem Grade gestört, wodurch auch der Stoffwechsel gehemmt wird; umso mehr Säure wird nun gebildet; dies alles schränkt, zusammen wirkend, den $\mathrm{CO}_{2}$-Gehalt im höchsten Grade ein, wodurch beträchtliche Abnahme des Blut- $p \mathrm{H}$ verursacht wird. Ja im Grunde bewirkt die Abnahme den $\mathrm{CO}_{2}$-Gehalt auch Verminderung des Blut- $p H$. Die gemeinschaftliche Untersuchung von Is hik a w a und $\mathrm{Ki}$ berichtete, dass die Sauerstoffkapazität des Hämoglobins am grössten ist, wenn das Blut- $p \mathrm{H}$ der Lösung zwischen 7,0-7,2 liegt. Es müsste daraus folgen, dass die Verminderung des $p \mathrm{H}$ des anämischen Bluts nicht anderes als das Mittel ist, die Sauerstoffkapazität möglichst gross, d. h. möglichst passend, zu erhalten. Bei meinem Versuch erreichte diese auch allmählich fast den höchsten Wert mit der Annäherung des $p \mathrm{H}$ an den genannten Wert.

Nach der Bestinmung der Katalase und des Glutathions bei Anämie vermindert sich ihr absoluter Wert oft unter den bei normaler Erythrozytenzahl, aber der Katalaseindex und der Glutathionquotient nehmen beträchtlich zu. Bei der Beobachtung ihres Verlaufs bei Anämie wurde festgestellt, dass der Wert der Katalase und der des Glutathions mit der Erholung von der Anämie abnehmen. Das Blut strebt überhaupt, wie ich bemerkte, nach möglichst grosser Vermehrung des Glutathion- und Katalasewerts, trotz der geringeren Menge von Erythrozyten als Überträger dieser Substanzen. Wird dann etwa die Vermehrung der Katalase und des Glutathions des anämischen Bluts durch gleichzeitig vorhandene Myelozytose zufällig hervorgebracht? Bach-Kopássy bestimmte an Kaninchen, bei denen er toxische Anämie hervorgerufen hatte, die Katalase und das Glutathion und auch nebenbei mit dem Hämatokrit das Volum 
der Erythrozyten. Dabei war die relative Zunahme der Katalase und des Glutathions noch grösser als die des Hämatokrits. Hieraus schloss er, dass die Vermehrung der Katalase und des Glutathions keine Beziehung zur Myelozytose habe. Wenn man diese Folgerung anerkennt, so müsste man die Vermehrung des Glutathions und der Katalase irgend einem andern Mechanismus zuschreiben.

$\mathrm{Gabbe}^{70)}$ und $\mathrm{H}$ andovsky $\mathrm{y}^{(1)}$ stellten die Annabme auf, dass das Glutathion bei Sauerstoffmangel kompensatorisch arbeite. Warb u r ${ }^{72)}$ leugnete nicht vollständig Bildung von Wasserstoff'superoxyd bei biologischer Oxydation; Wieland $d^{\text {i3 }}$ teilte mit, dass die Steigerung der Atemfunktion und die Vermehrung der Superoxybildung parallel miteinander verlaufen.

\section{Schluss.}

Ich ziehe aus den Ergebnissen meiner vorliegenden Versuche und auf Grund der Versuche von Ishikaw a u. Ki und mir nachstehende Folgerung:

Die bei Anämie hervorgebrachte

1. Vermehrung der Blutkatalase,

2. Vermehrung des reduzierten Glutathions und

3. Veränderung des $\mathrm{CO}_{2}$-Gelialts und des Blut- $p \mathrm{H}$ sind biologische Erscheinungen, die die durch zu wenig. Hämoglobin verursachte mangelhafte Sauerstofflieferung zu kompensieren streben, und sie sind gleiche provisorisclse Vorgänge wie die anderen bei Anämie auftretenden Erscheinungen : Beschleunigung der Atmung und Pulsation, Ửbergang der Gewebsflüssigkeiten in die Blutbahn usw.

\section{Literaturen.}

(1) B o hr, Skand. Arch. Physiol., 1892, 3, 76.

(2) Pleseh, Zsehr. exp. Ther, 1909, 6, 518.

(3) Weizsäeker, Dtsch. Arch. klin. Med., 1911, 101, 198.

(4) Morawitz u. Denecke, Arch. exp. Path. u. Pharm., 1921, 91, 37.

(5) Fahru. Ronzone, Arch. Int. Med., 1922, 29, 331.

(6) Liljestrand u. Stenström, Acta med. Scand., 1925, 63, 130.

(7) Blalock u. Harrison, A meric. Journ. Physiol., 1027, 80, 157.

(8) Is hika wa n. Ki, Tohoku Igaku Zassi, 1935, 18297.

(9) In o ue, Nippon Naikagakkai Zassi, 1919, 13, 784.

(10) Gesell, Physiol. Rev., 1925, 5, 551.

(11) B ald, Americ. Journ. Physiol., 1927, 81, 222.

(12) Riegel, Journ. Biol. Chem., 1927, 74, 123.

(13) Hertzmann u. Gese1l, Proc. Soc. Exp. Biol. and Med., 1925, 22, 248. 
(14) J a k s ch, Zschr. klin. Med., 1888, 13, 350.

(15) Peiper, Virchows Arch., 1889, 116, 337.

(16) K ra u s, Arch. exp. Path. u. Pharm., 1890, 26, 186.

(17) R a m p f, Zentrabl. klin. Med., 1891, 12, 441.

(18) Moranczenska, Virchows Arch., 1896, 144, 127.

(19) Branden burg, Zsehr. klin. Med, 1898, 36, 267.

(20) W aldrogel, Dtsch. med. Wschr., 1900, 685.

(21) Zuntz, zit. nach M o h r, Zschr. exp. Path. u. Ther., 1905, 2, 444.

(22) Bieling, Biochem. Zschr., 1914, 60, 421.

(23) Milroy, Journ. Physiol, 1917, 51, 259.

(24) Cann o n, Journ. Americ. Med. Ass., 1918, 70, 526.

(25) Barsky n. Kah n, Areh. Int. Med, 1919, 23, 337.

(26) Penfield, Americ. Journ. Physiol., 1919, 48, 121.

(27) Lindemannu. Gelter, Arch. Int. Med., 1920, 26, 453.

(28) Barru. Peters, Journ. Biol. Chem., 1921, 45, 571.

(29) K u hlma n n, Dtsch. Arch. klin. Med., 1921, 45, 346.

(30) O daira, Tohoku Journ. Exp. Med, 1923, 4, 243.

(31) Ge sell, Americ. Journ. Physiol, 1923, 66, 5.

(32) Koehler, Brunquist u. Loevenhardt, Journ. Biol. Chem., 1925, 64, 313.

(33) Wils on u. J ohnst o n, Journ. Biol. Chem., 1930, 85, 727.

(34) Ne u ma n n, Dtsch. Arch. klin. Med., 1905, 137, 324.

(35) Ochi, Nippon Naikagakkai Zassi, 1919, 9, 337.

(36) van Thienen, Dtsch. Arch. klin, Med., 1920, 131, 113

(37) Nisse n, Zschr klin. Med., 1921, 92,1.

(38) K o r all u s, Dtsch. Arch. klin. Med., 1922, 139, 252.

(39) Levinger u. B a ch, Zschr. kliu. Med., 1922, 95, 87.

(40) Segall, Dtsch. Arch. klin. Med.. 1922, 138, 243.

(41) Tögel a. Czera nke, Wien. Arch. klin. Med., 1925, 9, 381.

(42) Bernstein, Biochem. Zschr., 1926, 179, 304.

(43) Berg, Kong. ges. inn. Med., 1929, 54, 697.

(44) Bode, Zschr. ges. exp. Med., 1931, 77, 556.

(45) Krüger, Zschr. ges. exp. Med., 1933, 88, 437.

(46) Bach u. Kopássy, Magy, orv, arch. 1933, 34, 44. Ref.: Ber. ges. Physiol., 1933, 73, 285.

(47) B a ch u. B a ch, Biochem. Zschr., 1931, 236, 174.

(48) G abbe, Klin. Wschr., 1928, 2077.

(49) Waels ch, Med. Klin., 1933, 29, 1166.

(50) Pla tt, Brit. Journ. Exp. Path., 1931, 12, 212.

(51) Valera, Vilar u. Dusmarco, C.r. Soc. Biol. Paris, 1932, 106, 835.

(52) Butterfield, Hoppe-S eyler's Zschr. physiol. Chem., 1909, 62, 173.

(53) Morawitz a. Röhmer, Dtsch. Arch. klin. Med., 1908, 94, 529.

(54) Masing u. Siebeck, Dtsch. Arch. klin. Med., 1910, 99, 138.

(55) In oue u. Oe hi, Tokio Igakkai Zassi, 1919, 34, 1191.

(56) Gabbe, zit. nach Rona, Praktikum der physiologischen Chemie, T. 2, Berlin $1929,170$.

(57) Vau Slykeu. Cullen, Journ. Biol. Chem., 1917, 30, 305; Van Slyke u. St a di ie, Journ. Biol. Chem., 1922, 52, 263.

(58) Michaelis, zit. nach Abderhalden, Fandbuch der biologischen Arbeitsmethoden, Abt. III, T. A, H. 3, Berlin-Wien 1922, 495.

(59) B a rcrof t, The respiratory function of the blood, Cambridge 1914, 294. 
(60) L ö w y, Zentralbl. med. Wiss., 1894, 45, 785.

(61) Stra uss, Zschr. klin. Med., 1896, 30, 317.

(62) Evans, Brit. Journ. Fxp. Path., 1921, 2, 105.

(63) S a it o, Fukuoka Ikadaigaku Zassi, 1920, 13, 308.

(64) Fitzgerald, Journ. Path. Bact., 1910, 14, 328.

(65) T a tu m, Journ. Biol. Chem., 1920, 41, 59.

(66) Bennet, Journ. Biol. Chem., 1926, 69, 6075.

(67) Means, Bock u. Woodwell, Journ. Exp. Med., 1921, 33, 201.

(68) Henders o n u. Haggard, Journ. Americ. Med. Ass., 1922, 78, 697.

(69) Allen u. Page, Arch. exp. Path. u. Pharm., 1930, 147, 211.

(70) G a b be, Americ. Journ. Physiol., 1929, 90, 354. u. Zschr. ges. exp. Med., 1930, 69,392 .

(71) Handovsky, Klin. Wschr., 1930, 937.

(72) W a r bu rg, Ergebn. Physiol., 1914, 14, 253; Biochem. Zschr., 1923, 136, 226.

(73) Wiela nd, Ergebn. Physiol., 1922, 20, 477. 